\title{
Enhancement of Polyethylene Terephthalate Bottles Recycling in Mauritius Through the Creation of a Mobile Application
}

\author{
By Karishma Jankee ${ }^{1}$
}

\begin{abstract}
One million plastic bottles is currently consumed every minute in the world. This consumption trend is expected to further increase thereby worsening the problem of plastic pollution across the globe. Mauritius is also not left unaffected by plastic pollution and an increase in consumption of plastic bottles. Although plastic recycling is encouraged on the island, low recycling rate has been recorded $(40 \%)$. In this context, this research attempts to enhance the solid waste management methods of Mauritius, more specifically in terms of PET beverage bottles, by creating a persuasive technological tool that promotes recycling behavior among the citizens of Mauritius. The persuasive technological tool developed is a mobile application which allows its users to locate their nearest recycling bins while also providing them with facts about the effects and significance of plastic pollution and recycling respectively. The tool also included a survey page for gathering data on demographic, the recycling attitude/ behavior of the users prior to and after the introduction of the tool and on the functionalities of the tool itself. The results showed that the tool helped $80 \%$ of the users in initiating their recycling activities while the rest reported that the tool helped them increase those activities.
\end{abstract}

Keywords: Plastic pollution; Recycling; Persuasive technology; Pro-recycling attitude/behavior

\section{Introduction}

More than 500 billion plastic bottles are expected to be sold across the globe by 2021 due to the proliferation of an industrialized and a 'throw-away' culture. Moreover, non-refillable PET (polyethylene terephthalate) bottles come in all sizes, are convenient, lightweight, cheap, strong and act as hygienic containers for beverages which make them attractive to end users (Economisti Associati srl; BKP Development, 2015). Unfortunately, the existing waste management efforts and techniques are not adequate to match this current increasing consumption rate. It has also been found that the majority of plastic bottles produced in the 1940s is still present in our environment mainly due to its petrochemical based component within it (Zheyan, et al., 2017). In 2016, less than half of plastic bottles consumed had been collected for recycling purposes and only $7 \%$ were converted to new bottles while the rest ended up in either a landfill or in the sea. This in turn will eventually endanger our environment, our current generation and our future generations (Laville \& Taylor, 2017). This is due to the numerous environmental externalities associated with the use of PET bottles, for example, negative effects on human and ecosystem health, contamination of resources such as water and soil and aesthetic issues through littering on beaches among others (Economisti Associati srl; BKP 
Development, 2015). These externalities could be as devastating as climate change according to Laville \& Taylor (2017).

In Mauritius, plastic is the second largest contributor to the landfill waste with $12.4 \%$ and it contributes $15 \%$ of the total domestic waste of Mauritians (Ministry of Social Security, National Solidarity, and Environment and Sustainable Development, n.d.). Mauritius consumes approximately 90 to 100 million PET bottles annually. Out of the latter number of PET bottles consumed, only $40 \%$ is recycled while most of the remaining percentage end up in the sole landfill (Economisti Associati srl; BKP Development, 2015). Solid waste management in Mauritius is also becoming a complex issue to tackle since the Mare Chicose landfill is expected to reach its maximum capacity in 2018/19. Moreover, it costs approximately Rs 1 billion per year to the government to manage such waste, half of which is solely allocated to waste collection services (Ministry of Social Security, National Solidarity, and Environment and Sustainable Development, n.d.). In the attempt to resolve the issue of solid waste and its associated high cost, the government has made a tax of Rs 2 per PET bottle mandatory for bottlers and has successfully collected around Rs 190 million in 2014 through this levy. However, there is still approximately $27 \%$ of PET bottles which are not subjected to such tax (Economisti Associati srl; BKP Development, 2015). Although the government's attempt at tackling this issue at the bottlers level is commendable, there is also a need to engage individuals at community level to reduce littering as consumers are not necessarily encouraged to restrict their PET bottles consumption due to the convenience that these bottles provide and due to the lack of awareness on the several negative environmental externalities caused by PET bottles (University of Georgia, 2011; Economisti Associati srl; BKP Development, 2015). Moreover, there are several barriers to recycling at individual level due to the absence of dedicated and segregated waste collection system at household level, lack of recycling facilities such as segregation bins at home, lack of time for segregation of waste and transportation to relevant bins, lack of awareness of location of nearest recycling bins, lowincome level, discrepancy between attitude and behavior, lack of motivation due to perception that it is the responsibility of governments and large companies to tackle this issue among others (Anderson, et al., 2013; Economisti Associati srl; BKP Development, 2015; Mozo-Reyes, et al., 2016; Roan, 2017; Zheyan, et al., 2017).

Also, according to the Theory of Planned Behavior, an individual takes a behavioral decision based on expected benefits and costs while other factors such as attitude, subjective norm and perceived behavior control may influence the behavioral decision (Roan, 2017). Therefore, changing a behavior and promoting eco-friendly behavior may be a challenging attempt due to the range of factors and barriers which should be taken into consideration (Mozo-Reyes, et al., 2016; Roan, 2017). However, the engagement of everyone towards pro-recycling behavior could lead to a circular economy for plastic bottles which would in turn lead to saving money which could be used in other social and environmental projects while also reducing the consumption of fossil fuel (Laville \& Taylor, 2017). Consequently, easy, innovative, persuasive and attractive recycling methods should be developed to encourage the culture of recycling among individual citizens. This study, encourages and evaluates pro-recycling attitudes and behaviors of individuals through persuasive technology, which is a mobile application allowing its end users to locate their nearest recycling bins while providing positive cues for engaging in recycling activities. 


\section{Methodology and Data Collection}

This experimental study involved the development of a mobile application called 'My Recycling Guide' to assist users in locating their nearest recycling bins to ease and improve the recycling process through a map page ('My Map Guide') indicating almost all recycling bins found in Mauritius which is available for public use. The latter mobile application also included a page called 'My Fact Guide' which aimed at raising awareness on the effects of plastic pollution worldwide and in the Mauritian context while also providing tips to decrease plastic footprint at an individual level.

Moreover, the mobile application included a page called 'My Survey Guide'. There were three questionnaires in the latter page namely 'My Survey', 'My Dashboard' and 'App Rating'. The first questionnaire was focused on demographic as well as the recycling attitude and behavior of the user before the use of the mobile application. The second questionnaire was focused on the recycling behavior after the use of the mobile application, that is, questions about the type of waste recycled and recycling bins used among others. Lastly, the third questionnaire focused on the functionalities and design of the mobile application itself. Questions from all three surveys were based on information retrieved from a literature review based on four main topics namely status and impact of PET waste, theories pertaining to attitudes and behaviors, persuasive technology and mobile application development.

Convenience sampling technique was used to gather primary data and the mobile application was promoted to employees of Phoenix Beverages Ltd. ${ }^{1}$ (Limonaderie Site) and through word of mouth advertising. Once the mobile application was launched on Google Play, the number of active users was monitored through a website called Google Play Developer Console. The data from the questionnaires would be then automatically stored in a website called Firebase and then transferred on excel spreadsheet for data analyses, specifically for descriptive statistical analyses.

\section{Results Analysis and Discussion}

Results from the demographic section of the first questionnaire, 'My Survey', showed that the majority of users of the mobile application were females with an overall percentage of 64\%. Kollmuss and Agyeman (2002) suggested that gender and level of education are the two main demographic factors which have an influence on proenvironmental attitude and behavior. Recent studies have shown that women are more susceptible to change and are more emotionally engaged towards environmental issues regardless of their level of education (Kollmuss \& Agyeman, 2002; Gifford \& Nilsson, 2014). Gifford and Nilsson (2014) attempted to explain the latter gender differences in terms of different predominant personality traits in women compared to men. The latter authors also suggested that the pro-environmental behavior displayed by women may be due to them being more socially responsible compared to men. However, earlier research

${ }^{1}$ A public company listed on the Stock Exchange of Mauritius, Phoenix Beverages Ltd is said to be the leading beverage company in Mauritius. They produce and sell international brands and are the authorised bottler of the products of The Coca-Cola Company (TCCC). The Limonaderie Site bottles products of TCCC, Suntory Beverage \& Food Europe as well as local beverages such as Eski. 
had conflicting opinions about the latter notion of women being more caring of the environment.

It was found that $82 \%$ of users had completed their tertiary studies while the remaining had completed their secondary education or had other qualifications. Kollmuss and Agyeman (2002) inferred that people who are exposed to education for a longer period have a comprehensive knowledge about environmental problems and therefore have higher awareness levels which in turn promotes pro-environmental attitude. However, they also pointed out that the latter comprehensive environmental knowledge and awareness may not necessarily translate into pro-environmental actions as there are several other interacting personal, social, cultural and economic factors which may affect proenvironmental behaviors. Additionally, it was found in Norway that the longer the period of education, the least concerned citizens were about their environment which is particularly applicable for students in the business and technology fields of education (Gifford \& Nilsson, 2014).

Half of the users' age was noted to be between 25 and 35 while $27 \%$ of users' age range was between 36 and 46. Only 5\% of the users were younger than 25 years while the remaining users were older than 46 years. It was found in previous studies that younger individuals exhibit less pro-environmental actions compared to older individuals. This could be due to the older generation being exposed to some important environmental events which the younger generation have not experienced. On the other hand, more recent studies have shown that younger people show more environmental concern than the older generation (Gifford \& Nilsson, 2014).

Results from the second section of the first questionnaire showed that $68 \%$ of the survey participants believed that recycling is important for the economy, the society as well as the environment. The rest believed that recycling is an important activity which contributes solely to environmental benefits. It can be inferred that $32 \%$ of users are not fully aware of all the benefits associated with recycling activities although $82 \%$ of users had completed their tertiary studies. This shows that having completed tertiary education does not necessarily mean that individuals have the required environmental knowledge and awareness and these results tally with the study conducted by Gifford \& Nilsson (2014) as mentioned previously. These authors further suggested that individuals who choose to continue their tertiary studies in the environmental field, already displayed environmental concern and knowledge for them to make such choices. Therefore, tertiary studies within the environmental field may increase environmental knowledge but the pro-environmental attitude may not be entirely dependent on the latter form of education. The results also show that environmental education is not included as a mandatory subject in all educational fields in Mauritius. The previous government of Mauritius had attempted an educational reform through the inclusion of Education for Sustainable Development (ESD) under the Maurice Ile Durable (MID) project which was launched in 2008. ESD aimed at assisting young Mauritians in making informed decisions by providing them with the necessary support to develop the required environmental knowledge, skills and attitude (Ministry of Environment \& Sustainable Development, 2011). Unfortunately, with a change in government in 2014, the MID project was not adopted by the current government.

Apart from environmental education, Kollmuss and Agyeman (2002) suggested that 
cultural and social factors play a significant role in shaping the awareness and therefore attitude and behavior of an individual. For example, if a population is exposed to nature, the individuals are more prone to have a desire to protect their environment. In fact, it has been observed that in Germany, the citizens associate their forests with a high cultural value due to them being previously affected by acid rain. They therefore understand the link between protecting their natural resources in order not to be affected by environmental problems which could be catastrophic for not only the environment but also the society and the economy.

Furthermore, $68 \%$ of users described the recycling process as rewarding while $5 \%$ and $4 \%$ of users described the process as either worthless or too complicated respectively. Also, 9 users identified the lack of recycling programs organized by the government as a barrier to recycling while 7 and four users described the barriers to recycling as the absence of sufficient number of recycling bins across the island and as being unaware of the location of recycling bins respectively. The rest suggested that barriers were linked to the inconvenience of the recycling process or to recycling bins being constantly full. Also, 15 users reported that they did not experience external pressure to engage in recycling activities compared to only 7 users who did experience external pressure.

It can be deduced from the aforementioned results that although the majority of users believed that recycling is rewarding, several of them also encountered numerous barriers during the latter process. Consequently, it can be assumed that although the motivation is present in almost all cases through the sentiment of reward, there are several institutional barriers which conflict with the personal motivation of users. It has been found that citizens of a country are less likely to engage in recycling if they are not provided with satisfactory institutional opportunities (Kollmuss \& Agyeman, 2002). For example, the barrier of insufficient recycling bins has been reported in studies as well. Mauritius needs approximately 65 additional recycling bins to cater for the whole population as previous studies mention that there should be one recycling bin made available for every 12,000 residents. It is also important to place those bins in strategic areas and not concentrated in one area. These may have led to the reported barrier of recycling bins being constantly full (Economisti Associati srl; BKP Development, 2015). Regarding survey participants who described recycling as worthless and inconvenient, it can be inferred that different types of motivation should be provided to different groups of the Mauritian population as some citizens may be motivated by intrinsic rewards but others require extrinsic rewards to engage in pro-environmental behaviors (Kollmuss \& Agyeman, 2002).

It can also be inferred that there is a lack of shared responsibility in the environmental field as most individuals believed that the main barrier to their recycling activities were the lack of programs organized by the government. As per Gifford and Nilsson (2014), the sense of responsibility is an important factor which helps in shaping pro-environmental behaviors. In Netherlands, that sense of responsibility was found to be strongly linked with making financial sacrifices for the benefit of the environment. This is mainly because the sense of responsibility is often associated with a sense of guilt and therefore when individuals experience the latter, they show more positive attitude and behaviors. Additionally, it is important to include all concerned stakeholders and promote a sense of shared responsibility within the environmental field.

Lastly, 21 users had reported that they recycled prior to completing the survey compared 
to one who had never recycled. Furthermore, out of 22 users who participated in the survey, five responded that they recycled on a regular basis and 9 carried out waste segregation at home. This shows an inconsistency as it is difficult to recycle if waste has not been segregated. There could be other factors such as users engaging in recycling activities only at the workplace and not translating the same behavior at home. According to Kormos and Gifford (2014), self-reported information which are obtained through surveys rely on the assumption that the information provided by the surveyee is accurately depicting their actual behavior/ attitude which is why not all studies find that self-reported data are acceptable and accurate. On the contrary, those data are often found to be exaggerated and misleading but in this case, self-reports were chosen as a method to gather information as it is difficult to observe and constantly monitor actual recycling behaviors of individuals.

The results in the second questionnaire, 'My Dashboard' showed that only three out of 22 users reported that they recycled during the period of 12.04.2018 to 30.04.2018. Out of the latter three users, only one recycled plastic bottles while the other two recycled paper/ carton materials. All three participants used recycling bins which were located within a Winner's supermarket. On the other hand, almost all users consumed PET bottles on a weekly basis except for two users who reported that they do not consume any PET bottles. In this sample, the average number of PET bottles used weekly is approximately four. Users 10 and 11 consumed the highest number of PET bottles with 20 and 15 PET bottles per week respectively. However, neither user 10 nor user 11 reported having recycled PET bottles during the two weeks monitoring period.

The above results therefore demonstrate a gap between attitude and behavior which is in accordance with several studies. Kollmuss and Agyeman (2002) suggested that attitude have an influence on behavioral intentions rather than on behavior itself. Behavioral intentions which determine particular actions are themselves influenced by two factors namely social pressure and knowledge on consequence of actions. As was previously noted in the first survey, only 7 out of 22 participants reported that they experience social pressure to engage in recycling activities. Of those 7 participants, only two reported that they recycled on a regular basis. Therefore, if the suggestion by Kollmuss and Agyeman (2002) is taken into account, it can be inferred that those users are not fully aware of the consequences of not engaging in recycling activities. Kollmuss and Agyeman (2002) explained the latter through direct compared to indirect experience. Since recycling activities do not have a direct impact on individuals, it is less likely to have a change in behavior in those individuals. Moreover, if social and cultural norms are pre-dominantly unsustainable, it is highly likely to have a large gap between attitude and behavior. MozoReyes, et al. (2016) also reported that there is a culture of "out of sight, out of mind" when it comes to litter in Mauritius. Consequently, it can be deduced that there is also a cultural problem associated with the pro-recycling behavior of participants. However, since the monitoring period was rather short (approximately two weeks), some participants may have stored their recyclable waste which they may have disposed of after the monitoring period. Lastly, in terms of consumption of PET bottles, it was reported in the literature that Mauritians consume about 75 PET bottles annually (Economisti Associati srl; BKP Development, 2015). If the results of average consumption in this study is taken into account, Mauritians are possibly consuming about 208 PET bottles annually. This shows 
the need to conduct another survey on the consumption trends of Mauritians with respect to PET bottles.

It was also noted that out of 22 users who completed the first survey, only 15 completed the third survey. All 15 participants reported that the persuasive tool was very helpful and was very easy to use. The responses for the other features were distributed. For example, in terms of enjoyment of the tool, the majority reported that they really enjoyed using the mobile application while three and two users reported that they enjoyed using the tool and their enjoyment were neutral respectively. The response for improvement in quality of life brought by the tool was similar to that of the enjoyment feature. In terms of the time saving functionality, 13 participants reported that the mobile application definitely helped save their time. From the aforementioned results, it can be deduced that the persuasive tool developed was easy to use and was helpful and as per Arteaga, et al. (2009), perceived usefulness and ease of use are important elements to consider in the design phase of a persuasive technology.

Additionally, none of the functionalities received a negative feedback and as per OinasKukkonen and Harjumaa (2008), one of the two important factors to consider when evaluating the success of a persuasive technology is in terms of its functionalities. The other important factor is the contents of the tool which will be discussed below. On the other hand, Tonglet, et al. (2004) suggested that pro-recycling attitude and behavior can be attained by overcoming the sentiment of inconvenience in terms of time and space which are often associated with the recycling process. The results of the third survey showed that the persuasive technology assisted the users in saving time. It can therefore be inferred that the mobile application may have helped overcome the obstacle of inconvenience in terms of time.

In terms of persuasiveness of the tool, 12 users reported that the mobile application helped them to initiate their recycling activities while three users reported that the mobile application helped them increase their recycling activities. No users reported that the persuasive technology made no difference to their recycling activities. All users agreed that the information provided to them through the tool helped raise their awareness levels in terms of plastic pollution both globally and locally and in terms of recycling. Hence, the other important factor, content, which determines the success of a persuasive technology has also been fulfilled since the users reported an improvement in their recycling activities and the facts provided to them helped raise their awareness. Nevertheless, as reported in the literature, a persuasive technology should be initially developed for a wide range of audience and the latter tool should be upgraded over time to cater for different categories of users in terms of their needs and personalities (Halko \& Kientz, 2010). Subsequently, the last section of the 'App Rating' survey was an open-ended question which required users to provide their general feedback about the mobile application along with any improvements they wished to bring to the application. Some of the feedback involved the importance of sensitization of users prior to launching the application and the possibility of adding more levels to build up the application further. Other comments related to the application were that the tool was innovative, user friendly, useful, paperless, easily accessible, easing the recycling process, enhancing awareness on location of recycling units around the island among others. 


\section{Limitations}

Different challenges were encountered during the development of the mobile application. For instance, the technical limitations of the developer of the application and the gaps in the literature did not allow for the addition of features which could have increased the effectiveness of persuasion and ease of use of the tool. Features which could have been added with better coding knowledge are the provision of reminders, to users of the application, to constantly engage in recycling activities and the map page of the application could have been improved to include the option where users can type in their location so that their route to the relevant recycling bins is already mapped out. Also, due to technical barriers, the application developed could only be functional with an internet connection and was applicable to android phones only. Lastly, due to lack of information in the literature on the perception of Mauritians on the barriers of recycling, the tool could not address all barriers. Those technical challenges consequently decreased the number of participants which could have participated in the study surveys. Therefore, the sample size obtained after the introduction of the tool was rather small. Moreover, time allocated for the surveys were short. Although the technique used to select the sample group and gather data were found to be appropriate for this type of study, there were several constraints to those techniques namely sampling error and bias associated with convenience sampling and doubtful validity of self-reported information respectively.

\section{Recommendations}

Firstly, several improvements can be brought to this experimental research. For instance, Moore (2005) argued that there are different stages of attitude and behavioral change and it takes approximately one year for behavioral changes to occur. Moreover, different time periods for effective results are associated with different aims namely, reinforcement of current behavior, changing a specific behavior or shaping a new behavior (Oinas-Kukkonen \& Harjumaa, 2008). Consequently, the length and the sample size of this type of study could be extended to decrease sample bias and to better evaluate the effects of the mobile application over time on shaping, changing or enforcing behaviors. Additionally, since the mobile application developed was for a broad audience, as it was recommended by the literature as a starting point, the latter tool can be upgraded to cater for specific groups of individuals. Thus, increasing the sample size and categorizing different groups of people within the sample size according to personality types and their current behavior can assist in developing different persuasive strategies to cater for all categories of individuals (Arteaga, et al., 2009).

Apart from taking into consideration personality types for developing distinct strategies, causes of specific environmental damaging behaviors should also be investigated and considered in either antecedent (before behavior) or consequent (after behavior) strategies (Steg \& Vlek, 2009). The perception of Mauritians on the different barriers (e.g., lack of knowledge, ability to make informed choices, costs and benefits of change, culture, social norms and availability of infrastructure among others) associated with recycling could also be studied and considered in the upgrade of the tool. Adequate attitude-behavioral measures could be assessed and defined based on the aforementioned factors to eliminate 
the doubtfulness of self-reported data (Kollmuss \& Agyeman, 2002; Steg \& Vlek, 2009). Also, as demonstrated in the discussion, having completed tertiary education does not necessarily translate into having the appropriate environmental knowledge. To add further value to this study, more specific questions about environmental education could be included in surveys to assess the linkages between having the appropriate knowledge and pro-recycling attitudes.

Several technical improvements can be brought to the mobile application namely, reminders and notifications features can be added to prompt users to engage in recycling activities. The tool can be developed for iPhones and the mandatory internet connection can be made optional. The map section of the tool could also be improved to match the functionalities similar to that provided by Google maps. A feature which allows different users to be connected with each other through the mobile application could be added to provide social support. Trends on recycling activities of the user can be made available to the user for them to be able to better track their activities and improve. Lastly, the scope of the mobile application can be further extended to cater for different types of recyclable materials.

In addition to the possibility of enhancing the value of this study, further research for the local context in terms of persuasive technology along with waste management could provide valuable insights on the potential solutions to the waste management issue in Mauritius more so since the sole landfill of the island has almost reached it full capacity (Economisti Associati srl; BKP Development, 2015). Those researches should take into consideration all three sustainability factors namely, environmental, societal and economic. Recycling as a form of waste management should also be further studied as Mozo-Reyes, et al. (2016) found that out of 139 research conducted on several environmental activities from a technological perspective, only three discussed recycling. The feasibility of implementing some of the numerous recycling methods (buy-back centers, deposit system and curbside collection system among others) which have already been established in other countries could be assessed for Mauritius (Economisti Associati srl; BKP Development, 2015; Zheyan, et al., 2017).

Further research is still needed to provide conclusive explanation on the root causes which create the gap between environmental knowledge and actual pro-environmental behavior more specifically linked to waste management (Kollmuss \& Agyeman, 2002). Determining the root causes of the aforementioned gap could assist in defining and narrowing down a set of significant predictors of pro-recycling behavior and can therefore assist in developing appropriate waste management strategies. Narrowing down those predictors are essential as there are a wide range of factors which could be considered ranging from personal (e.g. experience, knowledge and problem awareness, personality, values, opinions and view, sense of responsibility and control, emotional engagement with a specific place, demographic, perceived efforts associated with recycling in terms of time and space), social (e.g. beliefs, norms, culture, social class, proximity to environmentally damaged sites), economic, technology and infrastructural facilities among others (Tonglet, et al., 2004; Gifford \& Nilsson, 2014). However, the latter may require extensive studies and metaanalyses as those numerous sources of influences are often interlinked and interact with each other and therefore, determining a set of relevant predictors may become complex and challenging (Gifford \& Nilsson, 2014). Research could also be conducted to categorize 
the significant predictors according to different aims namely reinforcement, shaping or changing behavior.

There are several actions which can be taken to decrease the number of recycling barriers in Mauritius. Firstly, to address the lack of environmental problem awareness among the public, the current government could re-adapt the educational reform which was part of the Maurice Ile Durable Project which was in turn overseen by the previous government (Ministry of Environment \& Sustainable Development, 2011). The education reform should aim at raising awareness on environmental issues among students of all ages. Dedicated bins for the segregation of waste at schools could also be implemented to shape a recycling culture from a young age. Secondly, since studies have shown that there should be one recycling bin for every 12,000 residents, civil societies and non-governmental organizations as well as corporates can invest in providing and installing the 65 additional bins which is required to cater for the Mauritian population. Thirdly, although PET bottlers are subject to taxes on PET bottles, legal regulations can be implemented for individuals as well to allow a decrease in the consumption of PET bottles or an increase in the number of recycled PET bottles. This may in turn allow a decrease in the amount of litter and therefore a decrease in costs associated with clean up campaigns (Economisti Associati srl; BKP Development, 2015).

Similarly, it is crucial for the government to consider all stakeholders concerned with waste management when developing waste management campaigns and policies particularly for recycling. This is because, recycling is the type of waste management which requires the most human intervention and participation (Mavropoulos, et al., 2015). One of the most important stakeholders are the individual citizens. By considering them and their opinions, a sense of shared responsibility can be created which could lead to mass collaboration and coordinated actions (Gifford \& Nilsson, 2014; Midden, 2015). Also, in this digital era, it is important for the government to evolve from traditional campaign methods to innovative digitalized campaigns. This digitalization allows for an expansion in the capability to reach and connect different stakeholders for more active and real-time participation in waste management programs (Mavropoulos, et al., 2015).

\section{Conclusion}

The tool developed was a new concept in Mauritius which allowed individuals to locate their nearest recycling bins. As a starting point, the tool was built for a broad audience as was recommended by the literature. After the tool was introduced to the chosen sample audience, information gathered on the demographic and the attitude/ behavior of the users have been evaluated in terms of recycling activities prior to and after the introduction of the mobile application. 37 individuals downloaded the mobile application while only 22 users completed the first survey, three users completed the second survey and 15 users completed the third survey. These results are in accordance with the literature which mentioned the constraints associated with participatory approach studies. Most participants were women who had completed their tertiary education and most of the users were of less than 46 years of age. The demographic of the participants also corroborated with the research conducted as most studies have shown that educated and young people and women show more concern for the environment, hence their 
participation. The results of attitude and behavior of users were in accordance with the literature as well, as most participants reported experiencing a positive personal impact during recycling activities. However, it was also reported that due to existing recycling barriers, the recycling process was difficult. Moreover, the results demonstrated the lack of environmental knowledge of participants even though most had completed their tertiary studies.

The barriers addressed and considered, through the mobile application, were firstly the location of the bins and secondly raising environmental and recycling awareness among the participants. However, most participants reported that the main recycling barrier was the lack of recycling programs organized by the government. It was therefore deduced that there was a lack of a sense of shared responsibility within the Mauritian population as there are existing infrastructure to accommodate for recycling activities although those facilities are not sufficient. Additionally, it can be considered that the doubtful validity of selfreported information, as reported in the literature, was confirmed in this case as although 21 users had reported that they recycled prior to this study and five users said that they recycle on a regular basis, only three users had input their recycling data in the second survey. Lastly, the mobile application was evaluated in terms of its effectiveness based on a literature review and feedback from the users of the mobile application. The effectiveness of the mobile application could not have been fully assessed mainly due to the aforementioned challenges, limitations and the small sample size and short time period for collection of data. On the other hand, the functionalities were evaluated and a positive feedback was received for all the functionalities assessed namely, ease of use, and enjoyment of application, time saving feature, and helpfulness among others.

This paper has therefore contributed to a field which has not been previously studied in the local context, that is, the combination of persuasive technology and waste management. It has been inferred that this study has successfully assisted in the enhancement of recycling activities at individual level since all participants reported that the tool developed either helped them initiate or increase their recycling activities and all reported that their level of awareness was increased through this tool. On the other hand, taking into account the gaps in the literature, the tool being a new concept for the local context and technical limitations of the developer of the tool, different challenges were encountered throughout this research work which consequently led to the identification of different limitations as mentioned previously. Therefore, there are numerous areas within this field which require more research and actions from different stakeholders.

\section{Acknowledgments}

I would like to express my sincere gratitude to the scholars of the University of Technology, Mauritius and to my affiliated organization, Phoenix Beverages Ltd for their assistance during this study. My gratitude also goes to the community of coders and software development across the world for their support. 


\section{References}

Anderson, B., Romani, J., Wentzel, M. \& Phillips, H., 2013. Recycling Behavior among Urban South Africans: The Role of Race and Social Status, New Orleans: Population Studies Center.

Arteaga, M., Kudeki, M. \& Woodworth, A., 2009. Combating Obesity Trends in Teenagers through Persuasive Mobile Technology. ACM SIGACCESS Accessibility and Computing, Issue 94, pp. 17-25.

Economisti Associati srl; BKP Development, 2015. Investigation of option to reduce the impacts of Polyethylene Terephthalate (PET) bottles in Mauritius, Online: Economisti Associati.

Gifford, R. \& Nilsson, N., 2014. Personal and social factors that influence pro-environmental concern and behaviour: A review. International Journal of Psychology, 49(3), pp. 141-157.

Halko, S. \& Kientz, J., 2010. Personality and Persuasive Technology: An Exploratory Study on HealthPromoting Mobile Applications. Seattle, ResearchGate, p. 150-161.

Kollmuss, A. \& Agyeman, J., 2002. Mind the Gap: why do people act environmentally and what are the barriers to pro-environmental behavior?. Environmental Education Research, 8(3), pp. 239-260.

Kormos, C. \& Gifford, R., 2014. The validity of self-report measures of proenvironmental behavior: A metaanalytic review. Journal of Environmental Psychology, Volume 40, pp. 359-37

Laville, S. \& Taylor, M., 2017. A million bottles a minute: world's plastic binge 'as dangerous as climate change'. [Online] Available at: https://www.theguardian.com/environment/2017/jun/28/a-million-aminute-worlds-plastic-bottle-binge-as-dangerous-as-climate-change [Accessed 28 February 2018].

Mavropoulos, A., Anthouli, A. \& Tsakona, M., 2015. Mobile Applications \& Waste Management: Recycling, Personal Behavior, Logistics, ResearchGate: D-Waste Environmental Consultants Ltd.

Midden, C., 2015. Persuasive technology for a sustainable society, s.l.: Eindhoven University of Technology.

Ministry of Environment \& Sustainable Development, 2011. Maurice Ile Durable Consultative Workshops. [Online] Available at: http://mid.govmu.org/portal/sites/mid/file/final-WG5.pdf [Accessed 02 May 2018].

Ministry of Social Security, National Solidarity, and Environment and Sustainable Development, n.d. Solid Waste Management In Mauritius. [Online] Available at: http://environment.govmu.org/English /Pages/swmd/SWMD-Solid-Waste-In-Mauritius.aspx [Accessed 05 September 2017].

Moore, M., 2005. The Transtheoretical Model of the Stages of Change and the Phases of Transformative Learning: Comparing Two Theories of Transformational Change. Transformative Education, 3(4), pp. 394-415.

Mozo-Reyes, E., Jambeck, J., Reeves, P. \& Johnsen, K., 2016. Will they recycle? Design and implementation of eco-feedback technology to promote on-the-go recycling in a university environment. Resources, Conservation and Recycling, Volume 114, pp. 72-79.

Oinas-Kukkonen, H. \& Harjumaa, M., 2008. A Systematic Framework for Designing and Evaluating Persuasive Systems. Finland, Springer, p. 164-176.

Roan, E., 2017. A Case Study of Motivating Factors Related to Recycling in the Workplace, Nebraska: University of Nebraska.

Steg, L. \& Vlek, C., 2009. Encouraging pro-environmental behaviour: An integrative review and research agenda. Environmental Psychology, Volume 29, pp. 309-317.

Tonglet, M., Phillips, P. S. \& Read, A. D., 2004. Using the Theory of Planned Behaviour to investigate the determinants of recycling behaviour: a case study from Brixworth, UK. Resources, Conservation and Recycling, Volume 41, pp. 191-214.

University of Georgia, 2011. Impacts to Public Recycling from Psychological and Technological Stimulus. [Online] Available at: https://erefdn.org/impacts-to-public-recycling-from-psychological-andtechnological-stimulus/ [Accessed 05 September 2017].

Zheyan, T., Ming, P., Beleya, P. \& Veerappan, G., 2017. Enhancement of Recycling Efforts through the Use of Mobile Application: A New Approach. Journal of Humanities, Language, Culture and Business, 1(1), pp. 84-92. 